\title{
Almost Everywhere First-Return Recovery
}

by

\author{
Michael J. EVANS and Paul D. HUMKE
}

Presented by Czestaw BESSAGA

\begin{abstract}
Summary. We present a new characterization of Lebesgue measurable functions; namely, a function $f:[0,1] \rightarrow \mathbb{R}$ is measurable if and only if it is first-return recoverable almost everywhere. This result is established by demonstrating a connection between almost everywhere first-return recovery and a first-return process for yielding the integral of a measurable function.
\end{abstract}

1. Introduction, notation, and definitions. We shall be considering real-valued functions defined on the interval $I \equiv[0,1]$. All references to integrals and measure are to Lebesgue integrals and Lebesgue measure. It was shown in [5] that a function $f: I \rightarrow \mathbb{R}$ belongs to Baire class one if and only if it is first-return recoverable everywhere. (Also see [1] for the result in a metric space setting, as well as [3], where numerous results involving the recovery of real functions on $I$ are examined.) Here we shall show that two other fundamental classes of real functions can be characterized in terms of first-return recovery if recovery everywhere is weakened to recovery except on a small set. In particular, we shall show (Theorem 2.3) that $f$ is measurable if and only if it is first-return recoverable except on a set of measure zero, and that $f$ has the Baire property if and only if it is first-return recoverable except on a set of first Baire category. We establish the measurable function result by first showing connections (Theorems 2.1 and 2.2) between the a.e. first-return recovery of $f$ and a first-return process for yielding the integral of $f$, as initially explored in [2]. To be more specific, we need to establish some notation and definitions.

2000 Mathematics Subject Classification: Primary 28A20, 26A42.

Key words and phrases: Lebesgue measurable functions, first-return recovery.

Work on this project began while the authors were Visiting Professors at the School of Mathematics and Statistics, University of St. Andrews, Scotland, and continued while Humke was a Visiting Professor at Washington and Lee University.

The authors wish to thank the referee for insightful comments and suggestions. 
Underlying all our subsequent definitions is the notion of what we call a trajectory on an interval $J \subset I$. A trajectory on $J$ is any sequence $\left\{x_{n}\right\}$ of distinct points in $J$ whose range is dense in $J$. If $J=I$ we usually refer to a trajectory on $I$ as simply a trajectory. (To see why we have opted for the term trajectory for such a sequence, the interested reader is referred to [4].) Any countable dense set $S \subset J$ is called a support set on $J$ and, of course, any enumeration of $S$ becomes a trajectory on $J$. For a given trajectory $\bar{x}=\left\{x_{n}\right\}$ and an interval $H$, we let $r(\bar{x}, H)$ denote the first $x_{n}$ that belongs to $H$. Finally, for $x \in[0,1]$ and $\varrho>0$ we let $B_{\varrho}(x)=\{y \in[0,1]:|y-x|<\varrho\}$.

Definition 1.1. Let $x \in I$ and let $\bar{x}=\left\{x_{n}\right\}$ be a fixed trajectory. The first-return route to $x, \mathcal{R}(\bar{x})_{x}=\left\{w_{k}(x)\right\}_{k=1}^{\infty}$, is defined recursively via

$$
w_{1}(x)=x_{0}, \quad w_{k+1}(x)= \begin{cases}r\left(\bar{x}, B_{\left|x-w_{k}(x)\right|}(x)\right) & \text { if } x \neq w_{k}(x), \\ w_{k}(x) & \text { if } x=w_{k}(x) .\end{cases}
$$

We say that $f$ is first-return recoverable with respect to $\bar{x}$ at $x$, or that $\bar{x}$ recovers $f$ at $x$, provided that

$$
\lim _{k \rightarrow \infty} f\left(w_{k}(x)\right)=f(x) .
$$

If this happens at every $x$ except for those in a measure zero set, we say that $f$ is first-return recoverable with respect to $\bar{x}$ a.e. Finally, we say that $f$ is a.e. first-return recoverable if there exists a trajectory $\bar{x}$ with respect to which $f$ is first-return recoverable a.e.

We shall use $\lambda(A)$ to denote the Lebesgue measure of a measurable set $A$.

Definition 1.2. Let $f: I \rightarrow \mathbb{R}$ be Lebesgue integrable. We say a trajectory $\bar{t}=\left\{t_{n}\right\}$ on I first-return yields the Lebesgue integral of $f$ (or simply that $\left\{t_{n}\right\}$ yields the Lebesgue integral of $f$ ) over a measurable set $A$ if for every $\varepsilon>0$ there is a $\delta>0$ such that

$$
\left|\sum_{J \in \mathcal{P}} f(r(\bar{t}, J)) \lambda(J \cap A)-\int_{A} f\right|<\varepsilon
$$

whenever $\mathcal{P}$ is a partition with $\operatorname{mesh}(\mathcal{P})<\delta$. We say that $\bar{t}$ integrates $f$ if $\bar{t}$ yields the Lebesgue integral of $f$ over every measurable set $A$.

Although it is not obvious from the definition, it is easy to see that if a trajectory yields the integral of a bounded measurable function $f$ over every closed interval, then that trajectory integrates $f$. That the same is true for arbitrary integrable functions is the subject of Lemma 2.3.

2. Results. We shall begin by establishing a fundamental link between Definitions 1.1 and 1.2. To do this we prove several preliminary lemmas.

Lemma 2.1. Let $\bar{x}=\left\{x_{n}\right\}$ be a trajectory and let $E$ denote the countable set $E=\left\{\left(x_{n}+x_{m}\right) / 2: n=0,1, \ldots ; m=0,1, \ldots\right\} \cup\{0,1\}$. Then for each 
$k=1,2, \ldots$ and for each $t \in I \backslash E$ there is an $\varepsilon>0$ such that $w_{k}$ is constant on $(t-\varepsilon, t+\varepsilon)$.

Proof. We shall prove this by induction on $k$. Since $w_{1}$ is constantly $x_{0}$ on $I$, the claim is true for $k=1$. Now, assume the claim is true for the natural number $k-1$. Let $t_{0} \in I \backslash E$. By the inductive hypothesis there is an $\varepsilon_{1}>0$ and a $y_{0} \in\left\{x_{n}\right\}$ such that $w_{k-1}(t)=y_{0}$ for all $t \in\left(t_{0}-\varepsilon_{1}, t_{0}+\varepsilon_{1}\right)$. Since $t_{0} \notin E$, we may assume the $\varepsilon_{1}$ is so small that $y_{0} \notin\left(t_{0}-\varepsilon_{1}, t_{0}+\varepsilon_{1}\right)$, and without loss of generality that $y_{0}<t_{0}-\varepsilon_{1}$. Set $c=t_{0}-\varepsilon_{1} / 2$ and $d=t_{0}+\varepsilon_{1} / 2$. Let $N=\min \left\{n: \bar{x}(n) \in\left(y_{0}, c\right)\right\}$.

Now, let $e=w_{k}(d)$ and $d_{1}=\left(y_{0}+e\right) / 2$. Then, clearly, $e=r\left(\bar{x},\left(y_{0}, 2 d-y_{0}\right)\right)$, and we readily observe that $w_{k}(t)=e$ for all $t \in\left(t_{0}-\varepsilon_{1}, t_{0}+\varepsilon_{1}\right) \cap\left(d_{1}, d\right]$. If it happens that $e=x_{N}$, then $d_{1}<c$ and, consequently, $w_{k}$ is constantly $e$ on $[c, d]$ and we are done in that case. Moreover, if it happens that $d_{1}<t_{0}$, then taking $\varepsilon=\min \left\{t_{0}-d_{1}, d-t_{0}\right\}$, we have $w_{k}$ constantly $e$ on $\left(t_{0}-\varepsilon, t_{0}+\varepsilon\right)$, and again we would be done. The remaining case is when $d_{1}>t_{0}$, and then $\bar{x}^{-1}(e)<N$. In this event, we repeat the above process, letting $d_{1}$ assume the role of $d$. That is, we set $e_{1}=w_{k}\left(d_{1}\right)$ and $d_{2}=\left(y_{0}+e_{1}\right) / 2$. Then, clearly, $e_{1}=r\left(\bar{x},\left(y_{0}, 2 d_{1}-y_{0}\right)\right)$, and we have $w_{k}(t)=e_{1}$ for all $t \in$ $\left(t_{0}-\varepsilon_{1}, t_{0}+\varepsilon_{1}\right) \cap\left(d_{2}, d_{1}\right]$. As before, if $e_{1}=x_{N}$, then $d_{2}<c$ and, consequently, $w_{k}$ is constantly $e_{1}$ on $\left[c, d_{1}\right]$ and we would be done. Furthermore, if it happens that $d_{2}<t_{0}$, then taking $\varepsilon=\min \left\{t_{0}-d_{2}, d_{1}-t_{0}\right\}$, we have $w_{k}$ constantly $e_{1}$ on $\left(t_{0}-\varepsilon, t_{0}+\varepsilon\right)$, and again we would be done. The remaining case is when $d_{2}>t_{0}$, in the event of which we would repeat the process again, defining $e_{3}=w_{k}\left(d_{2}\right)$ and $d_{3}=\left(y_{0}+e_{2}\right) / 2$. However, note that if we are in this case, then both $e$ and $e_{1}$ belong to $\left\{x_{0}, x_{1}, \ldots, x_{N-1}\right\}$. Thus this third case, resulting in our defining $e_{i}$ and $d_{i}$, can occur at most $N$ times. Thus, there must occur a stage at which either $e_{i}=x_{N}$ or $d_{i}<t_{0}$. In the former case we have $w_{k}$ constantly $e_{i}$ on $\left[c, d_{i-1}\right]$, indicating we are done. In the latter case, we may once again take $\varepsilon=\min \left\{t_{0}-d_{i}, d_{i-1}-t_{0}\right\}$ and conclude that $w_{k}$ is constantly $e_{i}$ on $\left(t_{0}-\varepsilon, t_{0}+\varepsilon\right)$, completing the proof.

Next, recall that a function belongs to class Baire* one provided every perfect set contains a portion (i.e., a relatively open interval) on which the function is continuous.

Lemma 2.2. Let $f: I \rightarrow \mathbb{R}$ be arbitrary and let $\bar{x}$ be a trajectory. For each $k$, the function $f \circ w_{k}: I \rightarrow \mathbb{R}$ belongs to class Baire* one.

Proof. Fix a $k \in \mathbb{N}$. From Lemma 2.1 we see that there exist countably many closed sets $T_{n}$ on each of which $w_{k}$ is constant such that $[0,1]=$ $\bigcup_{n=1}^{\infty} T_{n}$. Hence, $f \circ w_{k}$ is constant on each $T_{n}$, indicating that $f \circ w_{k}$ belongs to class Baire* one. 
LEMma 2.3. Let $f: I \rightarrow \mathbb{R}$ be integrable and let $\bar{x}$ be a trajectory such that $\bar{x}$ yields the integral of $f$ over every closed subinterval of $[0,1]$. Then $\bar{x}$ integrates $f$.

Proof. Let $\mathcal{G}=\{A \subset[0,1]: A$ is measurable, and $\bar{x}$ yields the integral of $f$ over $A\}$. Then $\mathcal{G}$ contains the null sets and, by hypothesis, the closed intervals. We show that $\mathcal{G}$ is an algebra and hence is all the measurable sets. To do this it suffices to show that $\mathcal{G}$ is closed under complementation and monotone unions.

- Complementation. Suppose $A \subset \mathcal{G}$. Then for any partition $\mathcal{P}$,

$$
\sum_{J \in \mathcal{P}} f \circ r(\bar{x}, J) \lambda\left(J \cap A^{\mathrm{c}}\right)=\sum_{J \in \mathcal{P}} f \circ r(\bar{x}, J) \lambda(J)-\sum_{J \in \mathcal{P}} f \circ r(\bar{x}, J) \lambda(J \cap A) .
$$

By hypothesis, as mesh $(\mathcal{P}) \rightarrow 0$ the right hand terms converge to $\int_{[0,1]} f$ and $\int_{A} f$ respectively and so the left hand term converges to $\int_{A^{\mathrm{c}}} f$.

- Monotone unions. Suppose $\bar{x}$ yields the integral of $f$ on $A_{n}$ and that $A_{n} \subset A_{n+1} \subset[0,1]$ for $n=1,2, \ldots$ We prove that $\bar{x}$ yields the integral of $f$ on $A \equiv \bigcup_{n=1}^{\infty} A_{n}$. The case where $f$ is bounded follows directly from the equality

$$
\begin{aligned}
\sum_{J \in \mathcal{P}} f \circ r(\bar{x}, J) \lambda(J \cap A)= & \sum_{J \in \mathcal{P}} f \circ r(\bar{x}, J) \lambda\left(J \cap A_{n}\right) \\
& +\sum_{J \in \mathcal{P}} f \circ r(\bar{x}, J) \lambda\left(J \cap\left(A \backslash A_{n}\right)\right) .
\end{aligned}
$$

To prove the general case takes a bit more work. Suppose the lemma is not true and that there is an $\varepsilon_{0}>0$ and a sequence $\left\{\mathcal{P}_{i}\right\}$ of partitions of $[0,1]$ with $\operatorname{mesh}\left(\mathcal{P}_{i}\right) \rightarrow 0$ such that

$$
\left|\sum_{J \in \mathcal{P}_{i}} f \circ r(\bar{x}, J) \lambda(J \cap A)-\int_{A} f\right| \geq \varepsilon_{0}
$$

for each $i=1,2, \ldots$ Then for every $n$ and $i$ we have

$$
\begin{aligned}
\left|\sum_{J \in \mathcal{P}_{i}} f \circ r(\bar{x}, J) \lambda\left(J \cap\left(A \backslash A_{n}\right)\right)\right| \\
+\left|\sum_{J \in \mathcal{P}_{i}} f \circ r(\bar{x}, J) \lambda\left(J \cap A_{n}\right)-\int_{A_{n}} f\right|+\left|\int_{A \backslash A_{n}} f\right| \geq \varepsilon_{0} .
\end{aligned}
$$

Let $0<\varrho<1$ and fix $N_{0}$ such that $\left|\int_{A \backslash A_{N_{0}}} f\right|<\varrho \varepsilon_{0}$. Fix $i_{0}$ such that for $i \geq i_{0},\left|\sum_{J \in \mathcal{P}_{i}} f \circ r(\bar{x}, J) \lambda\left(J \cap A_{N_{0}}\right)-\int_{A_{N_{0}}} f\right|<\varrho \varepsilon_{0}$; hence, for $i \geq i_{0}$,

$$
\left|\sum_{J \in \mathcal{P}_{i}} f \circ r(\bar{x}, J) \lambda\left(J \cap\left(A \backslash A_{N_{0}}\right)\right)\right| \geq(1-2 \varrho) \varepsilon_{0} .
$$


As $f$ is integrable with respect to $\bar{x}$ on $[0,1]$, there is a $\delta>0$ such that if $\operatorname{mesh}(\mathcal{P})<\delta$, then

$$
\left|\sum_{J \in \mathcal{P}} f \circ r(\bar{x}, J) \lambda(J)-\int_{[0,1]} f\right|<\varrho \varepsilon_{0} .
$$

Fix $i_{0}$ such that $\operatorname{mesh}\left(\mathcal{P}_{i_{0}}\right)<\delta$ and define a refinement $\mathcal{P}_{0}$ of $\mathcal{P}_{i_{0}}$ as follows. For each $J \in \mathcal{P}_{i_{0}}$ let $J_{0}$ be a closed interval of length $\lambda\left(\left(A \backslash A_{N_{0}}\right) \cap J\right)$ contained in the interior of $J$ such that $r(J) \in J_{0}$. Then the closure of $J \backslash J_{0}$ is the union of two intervals, $J_{-1}$ and $J_{1}$. Let $\mathcal{J}\left(J_{k}\right)$ be a partition of $J_{k}$, $k=-1,1$, sufficiently fine that

$$
\left|\sum_{J \in \mathcal{J}\left(J_{k}\right)} f \circ r(\bar{x}, J) \lambda(J)-\int_{I_{k}} f\right|<\frac{\varrho \varepsilon_{0}}{2 M},
$$

where $M=\operatorname{card} \mathcal{P}_{i_{0}}$. Define

$$
\mathcal{P}_{0}=\left\{J_{0}: J \in \mathcal{P}_{i_{0}}\right\} \cup \bigcup_{\substack{J \in \mathcal{P}_{i_{0}} \\ k=-1,1}} \mathcal{J}\left(J_{k}\right) .
$$

$\operatorname{As} \operatorname{mesh}\left(\mathcal{P}_{i_{0}}\right)<\delta$

$$
\left|\sum_{J \in \mathcal{P}_{0}} f \circ r(\bar{x}, J) \lambda(J)-\int_{[0,1]} f\right|<\varrho \varepsilon_{0},
$$

but

$$
\begin{aligned}
&\left|\sum_{J \in \mathcal{P}_{0}} f \circ r(\bar{x}, J) \lambda(J)-\int_{[0,1]} f\right| \geq\left|\sum_{J_{0} \in \mathcal{P},} f \circ r\left(\bar{x}, J_{0}\right) \lambda\left(J_{0}\right)\right| \\
&-\sum_{J \in \mathcal{P}_{i_{0}}} \sum_{k=-1,1}\left|\sum_{J \in \mathcal{J}\left(J_{k}\right)} f \circ r(\bar{x}, J) \lambda(J)-\int_{J_{k}} f\right|-\left|\int_{\substack{\cup\left\{J_{0}: J_{0} \in \mathcal{P}_{i_{0}}\right\} \\
\geq(1-4 \varrho) \varepsilon_{0} .}} f\right|
\end{aligned}
$$

For $\varrho \leq 1 / 5,(1)$ and (2) are contradictory, thus completing the proof.

Lemma 2.4. Let $f: I \rightarrow \mathbb{R}$ be integrable and let $\bar{x}$ be a trajectory such that $\bar{x}$ integrates $f$. Then for every measurable set $A$ and $\varepsilon>0$ there is $a \delta>0$ such that if $C=\left\{J_{1}, \ldots, J_{n}\right\}$ is any collection of non-overlapping intervals satisfying $\lambda\left(J_{k}\right)<\delta$ for $k=1, \ldots, n$ and $\sum_{k=1}^{n} \lambda\left(J_{k} \cap A\right)<\delta$, then $\left|\sum_{k=1}^{n} f \circ r\left(J_{k}\right) \lambda\left(J_{k} \cap A\right)\right|<\varepsilon$.

Proof. Suppose not. Then there is an $\varepsilon_{0}>0$ for which no $\delta$ exists. Fix $0<\varrho<1$. As $\bar{x}$ integrates $f$ on $A$, there is a $\delta_{1}>0$ such that if $\operatorname{mesh}(\mathcal{P})<\delta_{1}$ then

$$
\left|\sum_{J \in \mathcal{P}} f \circ r(J) \lambda(J \cap A)-\int_{A} f\right|<\varrho \varepsilon_{0} .
$$


Moreover, there is a $\delta_{2}>0$ such that if $\lambda(A \cap H)<\delta_{2}$, then

$$
\left|\int_{A \cap H} f\right|<\varrho \varepsilon_{0}
$$

Select $\delta=\min \left(\delta_{1}, \delta_{2}\right)$. Then there is a collection of non-overlaping intervals, $C=\left\{J_{k}: k=1, \ldots, n\right\}$ with $\lambda\left(J_{k}\right)<\delta$ for all $k$ and $\sum_{k=1}^{n} \lambda\left(J_{k} \cap A\right)<\delta$ and yet

$$
\left|\sum_{k=1}^{n} f \circ r\left(J_{k}\right) \lambda\left(J_{k} \cap A\right)\right| \geq \varepsilon_{0} .
$$

Let $\left\{I_{k}: k=1, \ldots, n+1\right\}$ denote the closed intervals contiguous with $H=\bigcup_{k=1}^{n} J_{k}$. Fix $k=1, \ldots, n+1$. As $f$ is integrable on $I_{k}$, there is a partition $\mathcal{J}\left(I_{k}\right)$ with $\operatorname{mesh}\left(\mathcal{J}\left(I_{k}\right)\right)<\delta_{1}$ such that

$$
\left|\sum_{J \in \mathcal{J}\left(I_{k}\right)} f \circ r(J) \lambda(J \cap A)-\int_{A \cap I_{k}} f\right|<\frac{\varrho \varepsilon_{0}}{n+1} .
$$

Let $\mathcal{P}=\left(\bigcup_{k=1}^{n+1} \mathcal{J}\left(I_{k}\right)\right) \cup C$. Then $\mathcal{P}$ is a partition of $[0,1]$ with $\operatorname{mesh}(\mathcal{P})<\delta_{1}$ and so using (3) and (4) we obtain

$$
\begin{aligned}
\varrho \varepsilon_{0}> & \left|\sum_{J \in \mathcal{P}} f \circ r(J) \lambda(J \cap A)-\int_{A} f\right| \\
= & \left|\sum_{k=1}^{n+1} \sum_{J \in \mathcal{J}\left(I_{k}\right)} f \circ r(J) \lambda(J \cap A)+\sum_{J \in C} f \circ r(J) \lambda(J \cap A)-\int_{A \backslash H} f-\int_{A \cap H} f\right| \\
\geq & \left|\sum_{J \in C} f \circ r(J) \lambda(J \cap A)\right|-\left|\sum_{k=1}^{n+1}\left(\sum_{J \in \mathcal{J}\left(I_{k}\right)} f \circ r(J) \lambda(J \cap A)-\int_{A \cap I_{k}} f\right)\right| \\
& -\left|\int_{A \cap H} f\right| \\
\geq & \varepsilon_{0}-2 \varrho \varepsilon_{0} .
\end{aligned}
$$

For $\varrho \leq 1 / 3$ this is a contradiction.

Lemma 2.5. Let $f: I \rightarrow \mathbb{R}$ be integrable and let $\bar{x}$ be a trajectory such that $\bar{x}$ integrates $f$. Then for every measurable set $A$ and $\varepsilon>0$ there is a $\delta>0$ such that

$$
\left|\sum_{J \in \mathcal{P}} f \circ r(\bar{x}, J) \lambda(J \cap A)-\int_{A \cap \bigcup_{J \in \mathcal{P}} J} f\right|<\varepsilon
$$

for every collection $\mathcal{P}$ of non-overlapping intervals with $\lambda\left(A \backslash \bigcup_{J \in \mathcal{P}} J\right)<\delta$.

Proof. Suppose the lemma is false. Then there is an $\varepsilon_{0}>0$ such that for each $n$ there exists a collection $\mathcal{C}_{n}=\left\{J_{1}^{n}, \ldots, J_{N_{n}}^{n}\right\}$ of non-overlapping 
intervals with $\lambda\left(J_{k}^{n}\right)<1 / n\left(k=1, \ldots, N_{n}\right), \lambda\left(A \backslash H_{n}\right)<1 / n$, where $H_{n}=$ $\bigcup_{k=1}^{N_{n}} J_{k}^{n}$, and yet

$$
\left|\sum_{k=1}^{N_{n}} f\left(r\left(J_{k}\right)\right) \lambda\left(J_{k}^{n} \cap A\right)-\int_{A \cap H} f\right| \geq \varepsilon_{0} .
$$

Fix $0<\varrho<1$ and choose $\delta>0$ so that

1. if $\operatorname{mesh}(\mathcal{P})<\delta$, then $\left|\sum_{J \in \mathcal{P}} f(r(J)) \lambda(J \cap A)-\int_{A} f\right|<\varrho \varepsilon_{0}$;

2. the conclusion of Lemma 2.4 holds for $\varepsilon=\varrho \varepsilon_{0}$;

3. $\left|\int_{A \cap E} f\right|<\varrho \varepsilon_{0}$ whenever $\lambda(A \cap E)<\delta$.

Fix $n>1 / \delta$ and "extend" $\mathcal{C}_{n}$ to a partition $\mathcal{P}$ of $[0,1]$ with $\operatorname{mesh}(\mathcal{P})<\delta$. Let $\mathcal{P}^{\prime}=\mathcal{P} \backslash \mathcal{C}_{n}$. Then

$$
\begin{aligned}
\varrho \varepsilon_{0} & >\left|\sum_{J \in \mathcal{P}} f(r(J)) \lambda(J \cap A)-\int_{A} f\right| \\
& =\left|\sum_{J \in \mathcal{C}_{n}} f(r(J)) \lambda(J \cap A)+\sum_{J \in \mathcal{P}^{\prime}} f(r(J)) \lambda(J \cap A)-\int_{A \backslash H_{n}} f-\int_{A \cap H_{n}} f\right| \\
& \geq\left|\sum_{J \in \mathcal{C}_{n}} f(r(J)) \lambda(J \cap A)-\int_{A \cap H_{n}} f\right|-\left|\sum_{J \in \mathcal{P}^{\prime}} f(r(J)) \lambda(J \cap A)\right|-\left|\int_{A \backslash H_{n}} f\right| \\
& \geq \varepsilon_{0}-2 \varrho \varepsilon_{0}
\end{aligned}
$$

and for $\varrho \leq 1 / 3$ this yields a contradiction.

Theorem 2.1. Let $f: I \rightarrow \mathbb{R}$ be Lebesgue integrable and suppose that the trajectory $\bar{x}$ integrates $f$. Then $\bar{x}$ recovers $f(x)$ almost everywhere.

Proof. Fix $c>0$ and define

$$
B=\left\{x: \limsup _{n \rightarrow \infty} f\left(\omega_{n}(x)\right)>f(x)+c\right\} .
$$

To verify the theorem, it is enough to check that $\lambda(B)=0$. As Lemma 2.2 implies that $B$ is measurable, we need only check that if $F \subset B$ is closed and $f$ is continuous on $F$, then $\lambda(F)=0$. Suppose, to the contrary, that there is such an $F \subset B$ with $\lambda(F)>0$.

Let $\varepsilon>0$ and choose $0<\delta<\lambda(F) / 2$ such that Lemma 2.5 holds for the set $F$ and $c \varepsilon$. Suppose too that $\left|f(x)-f\left(x^{\prime}\right)\right|<c / 2$ whenever $x, x^{\prime} \in F$ and $\left|x-x^{\prime}\right|<\delta$. Let $\mathcal{J}$ be the family of all intervals $J=[x-h, x+h]$ such that $h<\delta, x \in F$ and $f \circ r(J)>f(x)+c$. Then $\mathcal{J}$ is a Vitali cover of the set $F$ so there is a finite disjoint collection $J_{1}, \ldots, J_{n} \in \mathcal{J}$ such that writing $H=\bigcup_{k=1}^{n} J_{k}$ we have $\lambda(F \backslash H)<\delta$. Denoting the center of $J_{k}$ by $x_{k}$ we have

$$
\int_{F \cap H} f=\sum_{k=1}^{n} \int_{F \cap J_{k}} f \leq \sum_{k=1}^{n}\left(f\left(x_{k}\right)+\frac{c}{2}\right) \lambda\left(F \cap J_{k}\right)
$$




$$
\begin{aligned}
& \leq \sum_{k=1}^{n}\left(f \circ r\left(J_{k}\right)-\frac{c}{2}\right) \lambda\left(F \cap J_{k}\right) \\
& =\sum_{k=1}^{n}\left(f \circ r\left(J_{k}\right)\right) \lambda\left(F \cap J_{k}\right)-\frac{c}{2} \sum_{k=1}^{n} \lambda\left(F \cap J_{k}\right) \\
& \leq \int_{F \cap H} f+c \varepsilon-\frac{c}{2} \lambda(F \cap H) .
\end{aligned}
$$

Hence, $\lambda(F \cap H)<2 \varepsilon$ and so $\lambda(F)<4 \varepsilon$. This completes the proof.

Our primary interest in Theorem 2.1 in this paper is as a step toward a characterization of measurable functions; however, it is worth noting that it sheds additional light on the integration investigation begun in [2]. There it was shown that if $f$ is integrable then there exists a trajectory $\bar{x}$ which first-return yields the integral. Naturally, there are often trajectories which fail to yield the integral either by yielding the wrong number or by not yielding a number at all. Theorem 2.1 produces a necessary condition that $\bar{x}$ must satisfy in order to be a candidate for a trajectory that will yield the integral. As our next theorem shows, this condition is both necessary and sufficient for bounded measurable functions. The converse of Theorem 2.1 is not true in general for unbounded functions. For example, for the function

$$
f(x)= \begin{cases}1 / \sqrt{x}, & x \in(0,1], \\ 0, & x=0\end{cases}
$$

it is easy to think of a trajectory $\bar{x}$ which recovers $f$ everywhere, yet $\bar{x}$ fails to yield the integral.

Theorem 2.2. Let $f: I \rightarrow \mathbb{R}$ be bounded and measurable. A trajectory $\bar{t}=\left\{t_{n}\right\}$ recovers $f$ almost everywhere if and only if $\bar{t}$ integrates $f$.

Proof. Of course, the "if" direction follows immediately from Theorem 2.1. Next, assume that the trajectory $\bar{t}=\left\{t_{n}\right\}$ recovers $f$ almost everywhere, and assume that $|f|$ is bounded by a constant $M$. Fix $\varepsilon>0$.

Since $f\left(w_{k}(x)\right) \rightarrow f(x)$ a.e., it follows from Egorov's Theorem that there is an $m$ and a set $A \subseteq[0,1]$ with measure $\lambda(A)>1-\varepsilon$ such that

$$
\left|f\left(w_{k}(x)\right)-f(x)\right|<\varepsilon \quad \text { whenever } k>m \text { and } x \in A \text {. }
$$

We fix this $m$ for the remainder of the proof.

Next, for each $\delta>0$, let

$$
B_{\delta}=\left\{x \in[0,1]:(\forall i \leq m)\left|x-w_{i}(x)\right| \geq \delta\right\} .
$$

Letting $C_{n}=\left\{x:\left\{w_{1}(x), \ldots, w_{m}(x)\right\} \subset\left\{x_{1}, \ldots, x_{n}\right\}\right\}$, we have $\lambda\left(C_{n}\right) \rightarrow 1$, and thus there is an $n$ such that $\lambda\left(C_{n}\right)>1-\varepsilon / 2$. Then, upon setting $\delta^{\prime}=\varepsilon /(2 n)$, we have

$$
\lambda\left(B_{\delta^{\prime}}\right)>1-\varepsilon
$$


We now have $\lambda\left(A \cap B_{\delta^{\prime}}\right)>1-2 \varepsilon$ and so by Luzin's Theorem there is a compact $F \subseteq A \cap B_{\delta^{\prime}}$ such that $\lambda(F)>1-3 \varepsilon$ and $f$ is continuous on $F$. We choose $\eta<\delta^{\prime}$ such that

$$
|f(x)-f(y)|<\varepsilon \quad \text { whenever } x, y \in F \text { and }|x-y|<\eta .
$$

Now consider a partition $\mathcal{P}$ of mesh less than $\eta$ and write

$$
\mathcal{P}_{0}=\left\{J \in \mathcal{P}: \lambda(J \cap F) \leq \frac{1}{2} \lambda(J)\right\}, \quad \mathcal{P}_{1}=\mathcal{P} \backslash \mathcal{P}_{0} .
$$

For $J \in \mathcal{P}_{0}$,

$$
\lambda(J)=\lambda(J \cap F)+\lambda(J \backslash F) \leq \frac{1}{2} \lambda(J)+\lambda(J \backslash F),
$$

and hence $\lambda(J) \leq 2 \lambda(J \backslash F)$. Consequently,

$$
\sum_{J \in \mathcal{P}_{0}} \lambda(J)<6 \varepsilon
$$

Next, we claim that

$$
\text { if } J \in \mathcal{P}_{1} \text { and } x \in J \cap F \text {, then }|f(x)-f(r(\bar{t}, J))|<2 \varepsilon \text {. }
$$

To see this, let $J=[a, b], y=r(J)=r(\bar{t}, J)$; we can find $x^{\prime} \in F \cap J$ which is closer to $y$ than to either $a$ or $b\left(\right.$ since $\left.\lambda(F \cap J)>\frac{1}{2} \lambda(J)\right)$. Then $y=w_{k}\left(x^{\prime}\right)$ for some $k$, where $k>m$ by (6). Using (5), this yields $\left|f(y)-f\left(x^{\prime}\right)\right|<\varepsilon$ and we get $|f(y)-f(x)|<2 \varepsilon$ via $(7)$.

Using (8) and (9) we obtain

$$
\left|\sum_{J \in \mathcal{P}} f(r(J)) \lambda(J \cap F)-\int_{F} f\right| \leq \sum_{J \in \mathcal{P}} \int_{J \cap F}|f(r(J))-f| \leq 12 M \varepsilon+2 \varepsilon .
$$

In turn,

$$
\left|\sum_{J \in \mathcal{P}} f(r(J)) \lambda(J \cap F)-\int_{F} f\right| \text { and }\left|\sum_{J \in \mathcal{P}} f(r(J)) \lambda(J)-\int_{I} f\right|
$$

differ by at most $2 M \lambda\left(F^{\mathrm{c}}\right) \leq 6 M \varepsilon$, and hence

$$
\left|\sum_{J \in \mathcal{P}} f(r(J)) \lambda(J)-\int_{I} f\right|<18 M \varepsilon+2 \varepsilon .
$$

Since the above argument can clearly be applied to any subinterval $H$ of $I$, we see that $\bar{t}$ yields the Lebesgue integral of $f$ over any subinterval of $[0,1]$. That $\bar{t}$ integrates $f$ now follows from Lemma 2.3.

Now, using Theorem 2.2, we can prove the following characterization of measurable functions.

Theorem 2.3. A function $f: I \rightarrow \mathbb{R}$ is measurable if and only if it is a.e. recoverable.

Proof. First, suppose that $f$ is a.e. recoverable; that is, $\left\{f \circ w_{k}(x)\right\} \rightarrow f$ a.e. The measurability of $f$ now follows from Lemma 2.2 . 
Conversely, suppose that $f$ is measurable. As a first case suppose that $f$ is bounded. From [2] we know that there is a trajectory $\bar{x}$ which yields the integral of $f$ over every interval and hence integrates $f$. Applying Theorem 2.2 we see that $\bar{x}$ recovers $f$ a.e.

Finally, if $f$ is measurable but not bounded, then $\arctan (f)$ is measurable and bounded. From the above, there is a trajectory $\bar{x}$ which recovers $\arctan (f)$ a.e. However, it is an easy matter to see that at each point $x$ where $\bar{x}$ recovers $\arctan (f), \bar{x}$ also recovers $f$. Thus, $\bar{x}$ recovers $f$ a.e., completing the proof.

The topological, or categorical, analogue of Theorem 2.3 is also true and, as one would expect, has an easier proof. Recall that a set $A$ is said to have the Baire property if $A=G \triangle P$, where $G$ is open and $P$ is first category and $\triangle$ denotes the symmetric difference, and that $f: I \rightarrow \mathbb{R}$ has the Baire property if $f^{-1}(U)$ has the Baire property for each open set $U$.

THEOREM 2.4. A function $f: I \rightarrow \mathbb{R}$ has the Baire property if and only if it is recoverable except at a first category set of points.

Proof. First, let $f: I \rightarrow \mathbb{R}$ and assume that a trajectory $\bar{x}$ in $I$ recovers $f(x)$ at every point in $I \backslash S$, where $S$ is a set of first category; that is, $\left\{f \circ w_{k}(x)\right\} \rightarrow f(x)$ for each $x \in I \backslash S$. It follows from Lemma 2.2 that each $f \circ w_{k}$ has the Baire property, and since $\left\{f \circ w_{k}(x)\right\} \rightarrow f(x)$ except on a first category set, $f$ also has the Baire property.

Conversely, suppose that $f: I \rightarrow \mathbb{R}$ has the Baire property. Then there is a first category set $S$ such that the restricted function $\left.f\right|_{I \backslash S}$ is continuous. (See [6, p. 306] or [7, p. 36].) We may assume that $I \backslash S$ has no isolated points. Let $D$ be any support set lying entirely in $I \backslash S$ and let $\bar{x}$ be any ordering enumeration of $D$. Then, clearly, $\bar{x}$ recovers $f$ at every point of $I \backslash S$.

\section{References}

[1] U. B. Darji and M. J. Evans, Recovering Baire 1 functions, Mathematika 42 (1995), $43-48$.

[2] - - - A first return examination of the Lebesgue integral, Real Anal. Exchange 27 (2001/2002), 573-581.

[3] U. B. Darji, M. J. Evans, C. Freiling, and R. J. O'Malley, Fine properties of Baire one functions, Fund. Math. 155 (1998), 177-188.

[4] U. B. Darji, M. J. Evans, and R. J. O'Malley, First return path systems: differentiability, continuity, and orderings, Acta Math. Hungar. 66 (1995), 83-103.

[5] - - - - A first return characterization for Baire 1 functions, Real Anal. Exchange $19(1993 / 94), 510-515$.

[6] C. Kuratowski, Topologie, Vol. 1, 4th ed., Monograf. Mat. 20, PWN, Warszawa, 1958. 
[7] J. C. Oxtoby, Measure and Category. A Survey of the Analogies Between Topological and Measure Spaces, 2nd ed., Grad. Texts in Math. 2, Springer, New York, 1980.

Michael J. Evans

Department of Mathematics

Washington and Lee University

Lexington, VA 24450, U.S.A.

E-mail: mjevans@wlu.edu
Paul D. Humke

Department of Mathematics

St. Olaf College

Northfield, MN 45701, U.S.A.

E-mail: humke@stolaf.edu

Received March 20, 2004;

received in final form April 21, 2004 